\title{
ORIGINAL
}

\section{RETRASO DIAGNÓSTICO Y ENFERMEDAD AVANZADA EN LA INFECCIÓN POR EL VIRUS DE LA INMUNODEFICIENCIA HUMANA EN LA COMUNIDAD DE MADRID (2007-2011)}

\author{
Carlos Cevallos García, José Verdejo Ortés, Susana Martínez Rodríguez y Concepción Izarra \\ Pérez.
}

Servicio de Epidemiología. Consejería de Sanidad. Comunidad de Madrid

No existen conflictos de interés

\section{RESUMEN}

Fundamentos: El diagnóstico precoz de la infección por VIH conlleva un beneficio a nivel individual y de salud pública. El objetivo fue determinar la prevalencia de retrasos en el diagnóstico de infección por VIH en la Comunidad de Madrid (CM) y factores asociados.

Métodos: Estudio descriptivo de diagnósticos de VIH de enero2007 a septiembre-2011. Se estudiaron todos los nuevos diagnósticos con linfocitos CD4+ menores de 200/ $\mu$ l (presentación enfermedad avanzada, PEA), 350/ $\mu \mathrm{l}$ (retraso diagnóstico, RD) y 500/ $\mu 1$. Análisis descriptivo y multivariante mediante regresión logística.

Resultados: Se notificaron 3.347 casos. Se disponía de cifra de linfocitos CD4+ en 2.896 casos. El 29,7 presentaron PEA, 48,1\% RD y $67,7 \%<500 \mathrm{CD} 4+/ \mu$. Tanto en autóctonos como foráneos, la edad y mecanismo de transmisión (usuarios de drogas inyectadas (UDI) y heterosexual (HTX)) fueron variables independientes relacionadas con el diagnóstico tardío. En foráneos, el área geográfica de origen fue, igualmente, una variable independiente La probabilidad de PEA y RD en españoles aumentó con la edad de diagnóstico y fue mayor en HTX [OR:3,38 (IC95\%:2,29-4,98) y $2,44(1,67-3,56)]$ y UDI [OR:2,41 $(1,47-3,94)$ y $1,89(1,19-3,01)]$ respecto hombres que tienen sexo con hombres (HSH). La probabilidad de PEA y RD en foráneos aumentó con la edad de diagnóstico y fue mayor en HTX (OR:2,04 (1,44-2,89) y $2,29(1,61-3,25))$ respecto HSH y en procedentes de Latinoamérica [OR:2,56 (1,48-4,42) y $2,29(1,49-3,51)]$ y África Subsahariana [OR: $2,83(1,52-5,28)$ y $2,52(1,48-4,30)]$ respecto originarios de Europa Occidental.

Conclusiones: En la $\mathrm{CM}$ un elevado número de nuevos diagnósticos se producen en pacientes susceptibles a tratamiento, parte de ellos además presentan un grado importante de inmunosupresión. La edad, mecanismo de transmisión y país de origen se relacionan con un diagnóstico más tardío.

Palabras clave: VIH. Síndrome de inmunodeficiencia adquirida Diagnóstico tardío. Vigilancia epidemiológica.

Correspondencia

Carlos Cevallos García

C/ Julián Camarillo 4-B;

28037 Madrid

Teléfono 912052246

carlos.cevallos@salud.madrid.org

\section{ABSTRACT \\ Late Diagnosis of Human Immuno Deficiency Virus Infection in the Madrid Region (2007-2011)}

Background: Early HIV infection diagnosis means tremendous benefits both for the individual, in particular, and public health, in general. The aim is to analyze the prevalence of delayed HIV-infection diagnosis in Madrid region and its related factors.

Methods: Descriptive study of HIV-diagnosed patients from January 2007 to September 2011. A study was conducted on all new cases where the CD4+ count was below 200/ $\mu$ 1 ('presentation with advanced HIV disease', PAD), 350/ $\mu$ l ('late diagnosis', LD) and 500/ $\mu \mathrm{l}$. Descriptive and multivariate analysis by means of logistical regression.

Results: 3,347 HIV-diagnoses were reported, with CD4 count data available in 2,896 cases. $29.7 \%$ of which were PAD, $48.1 \%$ were LD and $67.7 \%<500$ CD $4+$. Both in Spaniards and foreigners, age and mode of transmission intravenous drug users (IDU) and heterosexual (HTX), were independent variables regarding late presentation. In foreigners, geographical origin was likewise an independent variable. In Spaniards the probability of contracting PAD and LD rise with age and was higher in HTX (OR:3.38 [95\%CI:2.29-4.98) and 2.44 (1.67-3.56)] and IDU [OR: 2.41 (1.47-3.94) and 1.89 (1.19-3.01)] than men who have sex with men (MSM). For their part, probability of PAD and LD in foreigners increased with age and was higher in HTX (OR: 2.04 [1.44-2.89) and $2.29(1.61-3.25)]$ than MSM and Latin American nationals (OR: 2.56 [1.48-4.42) and $2.29(1.49-3.51)]$ and Sub-Saharan Africans (OR: $2.83[1.52-5.28)$ and $2.52(1.48-4.30)]$ than Western Europeans.

Conclusions: In the Madrid Region a high number of new diagnoses are carried out in patients who should have already been under treatment. What is more some of them also present a significant degree of immuno-depression. Age, mode of transmission and geographical origin are closely related to late presentation.

Key words: HIV. Acquired Immunodeficiency Syndrome. Delayed diagnosis. Epidemiology. 


\section{INTRODUCCIÓN}

El diagnóstico precoz de los procesos patológicos, tanto agudos como crónicos, es generalmente determinante para la buena evolución de los mismos tanto a nivel individual como colectivo. A nivel individual es bien conocido que el pronóstico de muchos trastornos depende de la precocidad del diagnóstico, y a nivel colectivo la prevención secundaria, a través del diagnóstico temprano, juega un importante papel en salud pública. En este sentido, la infección por el virus de la inmunodeficiencia humana (VIH) es uno de los ejemplos más significativos, siendo su diagnóstico temprano un auténtico reto en salud pública si consideramos las consecuencias negativas derivadas de los retrasos diagnósticos ${ }^{1-5}$. El diagnóstico precoz de la infección por VIH permite un mejor manejo clínico de los pacientes instaurando el tratamiento antirretroviral (TAR) en el momento más adecuado, así como las pautas de quimioprofilaxis como prevención de infecciones oportunistas ${ }^{2-3}$. Así mismo, en términos de salud pública, permite un mejor control en la expansión de la enfermedad $^{4}$. Se sabe que la transmisión del VIH es mayor a partir de sujetos infectados que desconocen su estatus. Los pacientes que conocen su infección transmiten el VIH con menor frecuencia, ya que por una parte usan más medidas de barrera evitando transmisiones, y por otra parte, muchos de ellos presentan una carga viral indetectable al estar bajo TAR. Se estima que el riesgo de transmisión a partir de pacientes infectados no conocedores de su estatus es 3,5 veces superior al de los pacientes conocedores de su infección ${ }^{4}$. Existen numerosas publicaciones sobre el retraso diagnóstico, aunque no siempre las definiciones utilizadas han sido las mismas, lo que dificulta la comparación de los resultados. En numerosos trabajos han definido el retraso diagnóstico como aquel que se realiza cuando el paciente presenta un recuento de linfocitos CD4+ inferior a 200/ $\mu 1^{5-9}$. Recientemente se ha propuesto el límite de 350 linfocitos $\mathrm{CD} 4+/ \mu 1$ como nueva definición ${ }^{10-11}$.
En este artículo se analiza la prevalencia del retraso en el diagnóstico de infección por VIH durante el período comprendido entre el 1 de enero de 2007 y el 30 de septiembre de 2011 a partir de los casos notificados al Registro de Nuevos Diagnósticos de la Comunidad de Madrid (CM), así como sus factores asociados. Se han utilizado los criterios de definición que recientemente han sido propuestos ${ }^{10-11}$ : 'retraso diagnóstico' (RD) (linfocitos CD4+ al diagnóstico con valores inferiores de $350 / \mu \mathrm{l}$ ) y, el subgrupo, 'presentación con enfermedad avanzada' (PEA) (linfocitos CD4+ menores de $200 / \mu 1$ ). Adicionalmente, se ha estudiado la prevalencia de diagnósticos realizados con cifras de linfocitos CD4+ menores de 500/ $\mu 1$. Este límite de 500 células/ $\mu 1$, previamente comunicado $^{12-13}$ y previsiblemente aplicable en el futuro ${ }^{10}$, se justifica por dos hechos: a) cambios recientes realizados en las recomendaciones de TAR y b) la estrategia 'Test \& Treat'. Las recomendaciones desarrolladas por el Department of Health and Human Services (DHHS) en 2011 mantienen la recomendación de iniciar tratamiento en pacientes por debajo de 500 linfocitos CD4+/ $\mu 1^{14}$ Esta recomendación aparece en las recientes recomendaciones de la Sociedad Internacional de SIDA (International AIDS Society - USA Panel) de $2010^{15} \mathrm{y}$ en las recomendaciones del Ministerio de Sanidad de España de $2012^{16}$ Todo diagnóstico nuevo de infección por VIH debería hacerse cuando los pacientes todavía no presenten una disminución de linfocitos CD4+ por debajo de 500 células/ $\mu 1$ para obtener la máxima efectividad posible del tratamiento. Junto al beneficio individual, debe señalarse el que puede obtenerse en términos de salud pública. Basándose en la estrategia Universal Test and Treat, Charlebois ED et $\mathrm{al}^{17}$ han comunicado recientemente que la instauración de tratamiento antirretroviral a todos los pacientes con menos de 500 células/ $\mu 1$ reduciría en un $42 \%$ el número estimado de nuevas infecciones a los diez años.

El objetivo del estudio es determinar la prevalencia de 'retraso diagnóstico' y 'presentación con enfermedad avanzada' y los factores 
asociados en los nuevos diagnósticos de VIH notificados al Registro de VIH/sida desde enero de 2007 hasta septiembre de 2011 en la Comunidad de Madrid (CM). Adicionalmente se ha estudiado la prevalencia y factores asociados de diagnósticos de infección por VIH con menos de 500 linfocitos CD4+/ $\mu 1$, que establece el límite para iniciar TAR en las recomendaciones de la SPNS/GESIDA en España.

\section{SUJETOS Y MÉTODOS}

Se ha incluido como nuevo diagnósticos de VIH aquel realizado en una persona no diagnosticada previamente entre el 1 de enero de 2007 y 30 de septiembre de 2011, según criterios analíticos previamente establecidos en la Unión Europea ${ }^{18}$, notificados al Registro $\mathrm{VIH} /$ sida de la CM. La notificación de nuevos diagnósticos de infección por VIH en la CM es nominal y obligatoria para todos los profesionales sanitarios que realicen el diagnóstico, tratamiento y seguimiento de personas infectadas por el VIH ${ }^{19}$. El Servicio de Epidemiología de la CM recoge de forma exhaustiva aquellos diagnósticos de infección por VIH ocurridos desde el año 2007. Las variables recogidas incluyen: edad, sexo, mecanismo de transmisión, país de origen, recuento de linfocitos CD4+ y fecha de diagnóstico.

Se definió como 'retraso diagnóstico' (RD) todo sujeto que presentaba un recuento de linfocitos CD4+ inferior a 350/ $\mu$ l en el momento del diagnóstico de infección por VIH, y 'presentación con enfermedad avanzada' (PEA) todo sujeto que presenta una cifra inferior a 200 células/ $\mu 1$, además se estableció otro punto de corte en menos de 500 células/ $\mu$ l para el análisis .

Análisis estadístico. Se realizó análisis descriptivo de las variables recogidas. Se utilizó la prueba de chi-cuadrado o pruebas exactas para la comparación de variables cualitativas. Para evaluar los factores asociados a 'retraso diagnóstico' y a 'presentación con enfermedad avanzada' se hizo un análisis mul- tivariante con un modelo de regresión logística, obteniéndose la OR y su intervalo de confianza al 95\%. Para el análisis estadístico se utilizó el programa SPSS versión 18.0

\section{RESULTADOS}

Durante el período de estudio se notificaron 3.347 nuevos diagnósticos de infección por VIH. La media de edad en el momento del diagnóstico fue de 35,6 $\pm 10,7$ años (en hombres de $35,8 \pm 10,6$ y en mujeres $34,4 \pm 11,0$ años). El 83,7\% de los diagnósticos se dieron en varones (relación de 5,1:1). En lo que respecta a las conductas de riesgo en los hombres encontramos: hombres que tienen sexo con hombres (HSH): 1.860 (66,4\%), heterosexual (HTX): 410 (14,6\%), usuarios de drogas por vía intravenosa (UDI): $118(4,2 \%)$ y otros (transmisión por vía sexual sin especificar homo o heterosexual, transmisión vertical, trasfusión, desconocido, información no disponible): 413 (14,8\%). En las mujeres encontramos HTX: 480 (87,9\%), UDI: $28(5,1 \%)$ y otros (transmisión vertical, trasfusión, desconocido, información no disponible): 38 (7\%). Respecto a la zona geográfica de origen el 48,8\% habían nacido fuera de España. La distribución por regiones fue: América Latina y Caribe 1.003 (30\%), África subsahariana 330 (9,9\%), Europa Occidental 105 (3,1\%), Europa Oriental $130(3,9 \%)$, África del Norte y Oriente Medio $31(0,9 \%)$, América del Norte $16(0,5 \%)$, Asia $11(0,3 \%)$ y Oceanía 1 $(0,03 \%)$.

La población incluida en el estudio está constituida por los $2.896(86,5 \%)$ sujetos en los que se conocía el número de linfocitos CD4+. Sus características se muestran en la tabla 1.

Se apreciaron diferencias entre los pacientes con cifra de linfocitos CD4+ conocida con aquellos en lo que no constaba. En la distribución por sexos (mayor porcentaje de hombres en el grupo con información de CD4+: 84,2 vs 79,$8 ; \mathrm{p}=0,02$ ); en la distribución por mecanismo de transmisión, mayor porcentaje de $\mathrm{HSH}$ 
Tabla 1

Características de los nuevos diagnósticos de VIH con información de CD4 al diagnóstico. Comunidad de Madrid. Enero 2007- Septiembre 2011

\begin{tabular}{|c|c|c|c|}
\hline & España & Foráneos & Total \\
\hline \multicolumn{4}{|l|}{ SEXO } \\
\hline Varones $(\mathrm{n} / \%)$ & $1355(90,6 \%)$ & $1086(77,5 \%)$ & $2441(84,2 \%)$ \\
\hline Mujeres (n/\%) & $140(9,4 \%)$ & $315(22,5 \%)$ & $455(15,7 \%)$ \\
\hline Relación V/M & 9,7 & 3,4 & 5,3 \\
\hline \multicolumn{4}{|l|}{ EDAD } \\
\hline Media (DE) & $37,4(11,6)$ & $34,2(9,2)$ & $35,6(10,7)$ \\
\hline \multicolumn{4}{|c|}{ MECANISMO DE TRANSMISION* } \\
\hline HSH & $978(65,4 \%)$ & $711\left(50^{\prime} 7 \%\right)$ & $1689(58,3 \%)$ \\
\hline UDI & $87(5,8 \%)$ & $36(2,6 \%)$ & $123(4,2 \%)$ \\
\hline HTX & $232(15,5 \%)$ & $533(38,0 \%)$ & $765(26,4 \%)$ \\
\hline OTROS & $12(0,8 \%)$ & $4(0,3 \%)$ & $16(0,5 \%)$ \\
\hline Desconocido/NC & $186(12,5 \%)$ & $117(8,4 \%)$ & $303(10,4 \%)$ \\
\hline \multicolumn{4}{|c|}{ ÁREA GEOGRÁFICA DE ORIGEN } \\
\hline Europa Occidental & & $115(8,2 \%)$ & \\
\hline Europa Oriental & & $90(6,4 \%)$ & \\
\hline África Subsahariana & & $250(17,8 \%)$ & \\
\hline Norte de África y Oriente Medio & & $26(1,9 \%)$ & \\
\hline América del Norte & & $14(1,0 \%)$ & \\
\hline América Latina y Caribe & & $888(63,4 \%)$ & \\
\hline Asia & & $10(0,7 \%)$ & \\
\hline Oceanía & & $1(0,1 \%)$ & \\
\hline Desconocido & & $7(0,5 \%)$ & \\
\hline
\end{tabular}

*HSH: hombres que tienen sexo con hombres. UDI usuarios de drogas inyectadas. HTX: heterosexual

en sujetos con CD4+; 58,3 vs 37,9; $\mathrm{p}<0,001$ ); y en la distribución por área geográfica, mayor porcentaje de nacidos en África Subsahariana en el grupo sin CD4+: 34,2 vs 17,8 y mayor porcentaje de nacidos en América Latina en el grupo con CD4+: 63,4 vs 49,1; $\mathrm{p}<0,001$.

La mediana de la cifra de linfocitos CD4+ en el momento del diagnóstico fue de 364 células/ $\mu 1$ (rango intercuartílico 162-570). 860 $(29,7 \%)$ cumplieron la definición de 'presentación con enfermedad avanzada' (linfocitos CD4+<200), $1.394(48,1 \%)$ cumplieron la definición de 'retraso diagnóstico' (linfocitos

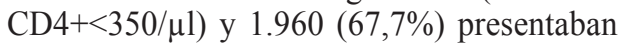
menos de 500 linfocitos $\mathrm{CD} 4+/ \mu 1$ en el momento del diagnóstico.
En conjunto, el RD y la PEA fue mayor en mujeres que en hombres (58\% vs 46,3\% para RD y $39,1 \%$ vs $27,9 \%$ para PEA), en sujetos heterosexuales $(64,1 \%$ para RD y 44,8 para PEA) respecto UDI $(57,7 \%$ RD y $41,5 \%$ PEA) y HSH (39, $7 \%$ RD y $21,4 \%$ PEA) y en foráneos $(53,9 \% \mathrm{RD}$ y $33,6 \% \mathrm{PEA})$ respecto a autóctonos (42,7\% RD y $26 \%$ PEA).

La prevalencia de diagnósticos de infección por VIH con menos de 500 linfocitos CD4+ al diagnóstico fue mayor en mujeres que en hombres $(75,8 \%$ vs $66,2 \%)$, en heterosexuales y UDI respecto a $\mathrm{HSH}(80,7 \%, 73,2 \%$ y $60,7 \%$ respectivamente) y también fue mayor en foráneos respecto autóctonos ( $73 \%$ vs $62,7 \%$ ). 
Tabla 2

Porcentaje de 'enfermedad avanzada' (linfocitos CD4+<200/ $\mu \mathrm{l}$ ), 'retraso diagnóstico' (linfocitos CD4 $+<350 / \mu \mathrm{l}$ ), diagnósticos con linfocitos CD4 $+<500 / \mu \mathrm{l}$ y factores asociados en personas nacidas en España. Comunidad de Madrid. 2007-2011

\begin{tabular}{|c|c|c|c|c|c|c|c|}
\hline & \multirow[b]{2}{*}{$\mathrm{n}$} & \multicolumn{2}{|c|}{$<200 / \mu 1$} & \multicolumn{2}{|c|}{$<350 / \mu l$} & \multicolumn{2}{|c|}{$<500 / \mu l$} \\
\hline & & $\%$ & $\begin{array}{l}\text { OR ajustado } \\
\text { (IC } 95 \% \text { ) }\end{array}$ & $\%$ & $\begin{array}{l}\text { OR ajustado } \\
\text { (IC 95\%) }\end{array}$ & $\%$ & $\begin{array}{c}\text { OR ajustado } \\
\text { (IC 95\%) }\end{array}$ \\
\hline \multicolumn{8}{|l|}{ SEXO } \\
\hline Hombre & 1.355 & 25,1 & $\begin{array}{c}1,48 \\
(0,92-2,37)\end{array}$ & 41,8 & $\begin{array}{c}1,28 \\
(0,81-2,01)\end{array}$ & 61,9 & $\begin{array}{c}1,25 \\
(0,75-2,09)\end{array}$ \\
\hline Mujer & 140 & 35,0 & 1,00 & 51,4 & 1,00 & 70,0 & 1,00 \\
\hline \multicolumn{8}{|c|}{ GRUPO DE EDAD } \\
\hline $0-19$ & 24 & 4,2 & \begin{tabular}{|c|}
0,74 \\
$(0,09-6,06)$
\end{tabular} & 8,3 & $\begin{array}{c}0,77 \\
(0,07-1,54)\end{array}$ & 25,0 & $\begin{array}{c}0,76 \\
(0,21-2,72)\end{array}$ \\
\hline $20-29$ & 374 & 10,7 & 1 & 27,3 & 1 & 52,7 & 1,00 \\
\hline $30-39$ & 541 & 20,1 & $\begin{array}{c}1,90 * \\
(1,28-2,83)\end{array}$ & 38,3 & $\begin{array}{c}1,53 * \\
(1,14-2,05)\end{array}$ & 58,6 & $\begin{array}{c}1,19 \\
(0,91-1,56)\end{array}$ \\
\hline $40-49$ & 352 & 37,8 & $\begin{array}{c}4,02 * \\
(2,68-6,02)\end{array}$ & 56,3 & \begin{tabular}{c|}
$2,85 *$ \\
$(2,07-3,92)$
\end{tabular} & 75,3 & $\begin{array}{c}2,06 * \\
(1,49-2,84)\end{array}$ \\
\hline$>49$ & 203 & 52,2 & $\begin{array}{c}6,92 * \\
(4,45-10,75)\end{array}$ & 63,5 & $\begin{array}{c}3,71 * \\
(2,55-5,41)\end{array}$ & 77,8 & $\begin{array}{c}2,54^{*} \\
(1,70-3,79)\end{array}$ \\
\hline \multicolumn{8}{|c|}{ MECANISMO DE TRANSMISIÓN } \\
\hline HSH & 978 & 18,4 & 1 & 35,2 & 1 & 56,5 & 1,00 \\
\hline UDI & 87 & 37,9 & $\begin{array}{c}2,41 * \\
(1,47-3,94)\end{array}$ & 54 & $\begin{array}{c}1,89 * \\
(1,19-3,01)\end{array}$ & 73,6 & $\begin{array}{c}1,96^{*} \\
(1,16-3,30)\end{array}$ \\
\hline HTX & 232 & 47,4 & \begin{tabular}{c|}
$3,38 *$ \\
$(2,29-4,98)$ \\
\end{tabular} & 61,2 & \begin{tabular}{c|}
$2,44 *$ \\
$(1,67-3,56)$ \\
\end{tabular} & 77,6 & $\begin{array}{c}2,37^{*} \\
(1,54-3,65)\end{array}$ \\
\hline $\begin{array}{c}\text { Otros/Desc } \\
\text { /NC }\end{array}$ & 198 & 33,3 & 1,92 & 53,5 & 1,95 & 70,7 & $\begin{array}{c}1,84 \\
(1,29-2,63)\end{array}$ \\
\hline
\end{tabular}

HSH: hombres que tienen sexo con hombres. UDI usuarios de drogas inyectadas. HTX: heterosexual; * $\mathrm{p}<0,01$

Se realizaron regresiones logísticas diferenciando españoles de sujetos procedentes de otros países, al encontrar interacciones entre el origen de los pacientes, la edad y modo de transmisión en el análisis multivariante. Los porcentajes encontrados de PEA, RD y diagnósticos de infección VIH con menos de 500 CD4+/ $\mu$ l en función de sexo, edad, mecanismo de transmisión y área de procedencia, así como el análisis multivariable se exponen en las tabla 2 y 3 .
La probabilidad de PEA en españoles fue mayor en los grupos de edad de 30-39 años (OR 1,90; IC95\% 1,28-2,83), 40-49 (OR 4,02; IC95\% 2,68-6,02) y mayores de 49 años (OR 6,92; IC95\% 4,45-10,75) respecto a los de 20-29 años de edad; y en HTX (OR 3,38; IC95\% 2,29-4,98) y UDI (OR 2,41; IC95\% 1,47-3,94) respecto a HSH. La probabilidad de PEA en foráneos fue mayor en los grupos de edad 30-39 años (OR 1,58; IC95\% 1,20-2,10), 40-49 años (OR 2,36; IC95\% 1,67-3,33) y >49 años (OR 2,32; IC95\% 1,46-3,67) respec- 
Tabla 3

Porcentaje de 'enfermedad avanzada' (linfocitos CD4+<200/ $\mu \mathrm{l}$ ), 'retraso diagnóstico' (linfocitos CD4 $+<350 / \mu \mathrm{l}$ ), diagnósticos con linfocitos CD4 $+<500 / \mu \mathrm{l}$ y factores asociados en personas nacidas fuera de España. Comunidad de Madrid. 2007-2011

\begin{tabular}{|c|c|c|c|c|c|c|c|}
\hline & \multirow[b]{2}{*}{$\mathrm{n}$} & \multicolumn{2}{|r|}{$<200 / \mu \mathrm{l}$} & \multicolumn{2}{|c|}{$<350 / \mu \mathrm{l}$} & \multicolumn{2}{|c|}{$<500 / \mu \mathrm{l}$} \\
\hline & & $\%$ & $\begin{array}{l}\text { OR ajustado } \\
\text { (IC } 95 \%)\end{array}$ & $\%$ & $\begin{array}{l}\text { OR ajustado } \\
\text { (IC } 95 \%)\end{array}$ & $\%$ & $\begin{array}{l}\text { OR ajustado } \\
\text { (IC } 95 \% \text { ) }\end{array}$ \\
\hline \multicolumn{8}{|l|}{ SEXO } \\
\hline Hombre & 1.086 & 31,5 & $\begin{array}{c}1,09 \\
(0,77-1,54) \\
\end{array}$ & 51,8 & $\begin{array}{c}1,28 \\
(0,89-1,84) \\
\end{array}$ & 71,5 & $\begin{array}{c}1,39 \\
(0,89-2,17) \\
\end{array}$ \\
\hline Mujer & 315 & 41 & 1 & 61 & 1,00 & 78,4 & 1,00 \\
\hline \multicolumn{8}{|l|}{ GRUPO DE EDAD } \\
\hline $0-19$ & 20 & 20 & $\begin{array}{c}0,76 \\
(0,23-2,46) \\
\end{array}$ & 45 & $\begin{array}{c}0,46 \\
(0,17-1,28)\end{array}$ & 45,0 & $\begin{array}{c}0,40 \\
(0,15-1,07)\end{array}$ \\
\hline $20-29$ & 479 & 25,9 & 1 & & 1,00 & 68,1 & 1,00 \\
\hline $30-39$ & 563 & 34,1 & $\begin{array}{c}1,58 * \\
(1,20-2,10) \\
\end{array}$ & 55,8 & $\begin{array}{c}1,47 * \\
(1,14-1,90) \\
\end{array}$ & 73,4 & $\begin{array}{c}1,31 \\
(0,99-1,73) \\
\end{array}$ \\
\hline $40-49$ & 238 & 44,1 & $\begin{array}{c}2,36 * \\
(1,67-3,33) \\
\end{array}$ & 60,9 & $\begin{array}{c}1,76 * \\
(1,26-2,46) \\
\end{array}$ & 79,4 & $\begin{array}{c}1,76^{*} \\
(1,19-2,59) \\
\end{array}$ \\
\hline$>49$ & 101 & 45,5 & $\begin{array}{c}2,32 * \\
(1,46-3,67) \\
\end{array}$ & 65,3 & $\begin{array}{c}1,94 * \\
(1,22-3,09) \\
\end{array}$ & 85,1 & $\begin{array}{c}2,40 * \\
(1,32-4,36) \\
\end{array}$ \\
\hline \multicolumn{8}{|c|}{ MECANISMO DE TRANSMISIÓN } \\
\hline $\mathrm{HSH}$ & 711 & 25,6 & 1 & 46 & 1,00 & 66,5 & 1,00 \\
\hline UDI & 36 & 50 & $\begin{array}{c}2,09 \\
(0,91-4,81) \\
\end{array}$ & 66,7 & $\begin{array}{c}2,62 * \\
(1,13-6,11) \\
\end{array}$ & 72,2 & $\begin{array}{c}2,00 \\
(0,83-4,86) \\
\end{array}$ \\
\hline HTX & 533 & 43,7 & $\begin{array}{c}2,04^{*} \\
(1,44-2,89) \\
\end{array}$ & 65,3 & $\begin{array}{c}2,29 * \\
(1,61-3,25) \\
\end{array}$ & 82,0 & $\begin{array}{c}2,80 * \\
(1,81-4,33) \\
\end{array}$ \\
\hline Otros/Desc./NC & 121 & 31,4 & $\begin{array}{c}1,38 \\
(0,87-2,20) \\
\end{array}$ & 46,3 & $\begin{array}{c}1,06 \\
(0,68-1,63) \\
\end{array}$ & 71,9 & $\begin{array}{c}1,36 \\
(0,84-2,20) \\
\end{array}$ \\
\hline \multicolumn{8}{|c|}{ ÁREA GEOGRÁFICA DE ORIGEN } \\
\hline Europa Occidental & 115 & 14,8 & 1 & 31,3 & 1,00 & 60,0 & 1,00 \\
\hline Europa Oriental & 90 & 38,9 & $\begin{array}{c}3,09 * \\
(1,50-6,37)\end{array}$ & 50 & $\begin{array}{c}1,65 \\
(0,87-3,12) \\
\end{array}$ & 63,3 & $\begin{array}{c}0,96 \\
(0,50-1,82) \\
\end{array}$ \\
\hline África Subsahariana & 250 & 44,4 & $\begin{array}{c}2,83^{*} \\
(1,52-5,28)\end{array}$ & 66 & $\begin{array}{c}2,52 * \\
(1,48-4,30)\end{array}$ & 80,8 & $\begin{array}{c}1,70 \\
(0,96-3,02) \\
\end{array}$ \\
\hline $\begin{array}{l}\text { Norte África Oriente- } \\
\text { Medio }\end{array}$ & 26 & 53,8 & $\begin{array}{c}4,62 * \\
(1,78-12,02)\end{array}$ & 73,1 & $\begin{array}{c}3,93^{*} \\
(1,48-10,44)\end{array}$ & 70,8 & $\begin{array}{c}2,02 \\
(0,69-5,92)\end{array}$ \\
\hline América del Norte & 14 & 35,7 & $\begin{array}{c}3,23 \\
(0,95-11,04) \\
\end{array}$ & 50 & $\begin{array}{c}2,24 * \\
(0,72-6,94)\end{array}$ & 71,4 & $\begin{array}{c}1,85 \\
(0,54-6,36)\end{array}$ \\
\hline América Latina Caribe & 888 & 31,3 & $\begin{array}{c}2,56^{*} \\
(1,48-4,42) \\
\end{array}$ & 53,0 & $\begin{array}{c}2,29^{*} \\
(1,49-3,51) \\
\end{array}$ & 73,2 & $\begin{array}{c}1,89^{*} \\
(1,24-2,86) \\
\end{array}$ \\
\hline Asia & 10 & 40,0 & $\begin{array}{c}3,32 \\
(0,79-14,01) \\
\end{array}$ & 50,0 & $\begin{array}{c}1,78 \\
(0,46-6,93) \\
\end{array}$ & 70,0 & $\begin{array}{c}1,34 \\
(0,31-5,83) \\
\end{array}$ \\
\hline
\end{tabular}

HSH: hombres que tienen sexo con hombres. UDI usuarios de drogas inyectadas. HTX: heterosexual; ${ }^{*} \mathrm{p}<0,01$ 
to a los de 20-29 años y en HTX (OR 2,04; IC95\% 1,44-2,89) respecto a HSH. Con respecto a las áreas geográficas, la probabilidad fue mayor en los sujetos procedentes de América Latina y Caribe (OR 2,56; IC95\% 1,48-4,42), África Subsahariana (OR 2,83; IC95\% 1,52-5,28), Europa Oriental (OR 3,09 ; IC95\% 1,50-6,37) y África del Norte y Oriente Medio (OR 4,62; IC95\% 1,78$12,02)$ en relación con Europa Occidental.

La probabilidad de RD en españoles fue mayor en los grupos de edad 30-39 años (OR 1,53; IC95\% 1,14-2,05), 40-49 (OR 2,85 ; IC95\% 2,07-3,92) y mayores de 49 años (OR 3,71; IC95\% 2,55-5,41); y en HTX (OR 2,44; IC95\% 1,67-3,56) y UDI (OR 1,89; IC95\% 1,19-3,01). La probabilidad de RD en foráneos fue mayor en los grupos de edad 30-39 años (OR 1,47; IC95\% 1,14-1,90), 40-49 (OR 1,76; IC95\% 1,26$2,46)$ y $>49$ años (OR 1,94; IC95\% 1,22$3,09)$; y en UDI (OR 2,62; IC95\% 1,136,11 ) y HTX (OR 2,29; IC95\% 1,61-3,25). Con respecto a áreas, la probabilidad fue mayor en los procedentes de África Subsahariana (OR 2,52; IC95\% 1,48-4,30), América Latina y Caribe (OR 2,29; IC95\% 1,49$3,51)$ y África del Norte y Oriente Medio (OR 3,93; IC95\% 1,48-10,44) en relación con Europa Occidental.

La probabilidad de que las personas españolas diagnosticadas de infección por VIH presenten menos de $500 \mathrm{CD} 4 * / \mu 1$ en el momento del diagnóstico fue mayor en los grupos de edad 40-49 años (OR 2,06; IC95\% 1,49-2,84) y mayores de 49 años (OR 2,54; OR95\% 1,70-3,79); y en HTX (OR 2,37; IC95\% 1,54-3,65) y UDI (OR 1,96; IC95\% 1,16-3,30). La probabilidad en foráneos fue mayor en los grupos de edad 40-49 años (OR 1,76; IC95\% 1,19-2,59) y $>49$ años (OR 2,40; IC95\% 1,32-4,36); y en HTX (OR 2,80; IC95\% 1,81-4,33). Con respecto a áreas geográficas, la probabilidad fue mayor en los procedentes de América Latina y Caribe (OR 1,89; IC95\% 1,24$2,86)$.

\section{DISCUSIÓN}

En este estudio hemos encontrado que la prevalencia de infecciones por VIH diagnosticadas con menos de 200 linfocitos CD4+/ $\mu$, menos de $350 \mathrm{CD} 4+/ \mu 1$ y menos de 500 $\mathrm{CD} 4+/ \mu \mathrm{l}$ ha sido de $29,7 \%, 48,1 \%$ y $67,6 \%$ respectivamente. Tanto el mecanismo de transmisión como la edad se han encontrado asociados al diagnóstico tardío en autóctonos y foráneos. El uso de drogas inyectadas, la transmisión heterosexual y la edad por encima de 30 años se relacionan con un mayor retraso en el diagnóstico.

La mediana de linfocitos CD4+ al diagnóstico es de $364 / \mu 1$. Althoff $\mathrm{KN}$ et $\mathrm{al}^{20}$, han valorado el recuento de linfocitos CD4+ desde 1997 hasta 2007 en 13 cohortes de Estados Unidos y Canadá. La mediana de linfocitos CD4+ en 1997 fue de 256 células/ $\mu 1$ y se fue incrementando hasta llegar a 317 células/ $\mu 1$ en 2007. Los autores consideran positivo este aumento de la mediana de linfocitos CD4+ ya que expresa una disminución en el tiempo transcurrido desde la infección hasta el diagnóstico. A pesar de la mejora, la mediana de linfocitos CD4 se sigue manteniendo por debajo de 350/ $\mu$ l. Sin embargo, recientemente, Diez $\mathrm{M}$ et $\mathrm{al}^{21}$ han comunicado una mediana al diagnóstico de $347 \mathrm{CD} 4+/ \mu 1$ en el año 2009 en 15 Comunidades Autónomas en España, incluyendo la CM. Las cifras que se han obtenido en nuestro estudio son superiores a las de Althoff ${ }^{20}$ y similares a las de Diez ${ }^{21}$.

En relación a la PEA, el $29,7 \%$ de los pacientes presentaban menos de 200 linfocitos $\mathrm{CD} 4+/ \mu 1$. En la literatura se han comunicado cifras que oscilan entre $24 \%$ y $43 \% 0^{5-7,}, 9,22$. Nuestro porcentaje es inferior al $37,3 \%$, publicado por J Oliva et $\mathrm{al}^{23}$ a partir de los datos obtenidos procedentes del Sistema de Información de Nuevos Diagnósticos de VIH (SINIVIH) de 8 Comunidades Autónomas de España en el período 2003-2007, esta cifra corresponde a un periodo anterior al de nuestro estudio y, además, teniendo en cuenta que este porcentaje mejora cada año según la 
investigación de Oliva J (42,7\% en 2003 y $33,4 \%$ en 2007).

En lo que respecta a los RD $(<350 \mathrm{CD} 4+/$ $\mu 1)$ nuestro porcentaje del $48,1 \%$ al compararlo con estudios en nuestro medio es similar a otros publicados recientemente ${ }^{21-22,24} \mathrm{e}$ inferior al $56,2 \%$ de Oliva $\mathrm{J}$ et $\mathrm{al}^{23}$, igualmente es inferior al 59,3\% comunicado por Sullivan AK et $\mathrm{al}^{9}$ En 2010, la cifra media de RD en la Unión Europea ha sido similar a la encontrada por nosotros. No obstante el rango encontrado entre sus diferentes países oscila entre el $25,3 \%$ de la República Checa y el 62,7\% de Portugal ${ }^{25}$.

El 67,7\% de los nuevos diagnósticos fueron realizados en pacientes con recuentos de linfocitos CD4+ por debajo de 500/ $\mu$ l. Solo un 32,3 $\%$ fueron diagnosticados en un momento óptimo para la valoración terapéutica. Recientemente, en la Comunidad de Aragón, se ha comunicado que dos tercios de los nuevos diagnósticos realizados en 2009 presentaban un recuento de linfocitos CD4+ inferior a $500 / \mu 1^{13}$. Este porcentaje es superponible al nuestro de 67,7\%. En Reino Unido e Irlanda casi el $80 \%$ de los pacientes diagnosticados en 2003 presentaban un recuento inferior a $500^{9}$.

Hemos encontrado que tanto el RD como la PEA se relacionan con la edad, el mecanismo de transmisión y el área geográfica de origen. No hemos observado un mayor riesgo en hombres, lo que contrasta con los hallazgos de $\mathrm{J}$ Oliva et $\mathrm{al}^{23}$. En relación con la edad, el $\mathrm{RD} / \mathrm{PEA}$ ha sido mayor en los pacientes con edades más elevadas. Hallazgos similares han sido comunicados en España ${ }^{22-23,26}$, Italia $^{8}$ y Estados Unidos ${ }^{27}$. Probablemente, al menos en parte se relaciona con una menor percepción de riesgo por parte de los pacientes y, quizás, por parte de los profesionales sanitarios.

En relación con el mecanismo de transmisión el RD/PEA es más frecuente entre las personas heterosexuales. Este hallazgo, ya comunicado previamente ${ }^{22-23,26}$, probablemente se relaciona con una percepción de riesgo menor de los sujetos heterosexuales. Los HSH tienen mayor percepción de riesgo y no es infrecuente que periódicamente se realicen pruebas diagnósticas. Esta mentalización no existe, en general, entre las personas heterosexuales. Así mismo, la sospecha diagnóstica de infección por VIH entre el personal sanitario es menor cuando se trata pacientes heterosexuales. En lo referente a sospecha diagnóstica, en el extremo opuesto se sitúan los UDI. Sin embargo, hemos observado un 57,7\% y $41,5 \%$ de $\mathrm{RD} / \mathrm{PEA}$ entre los UDI. Esta asociación ha sido previamente publicada en nuestro país ${ }^{22-}$ 23,26 . Es posible que un porcentaje no desdeñable de estos pacientes no sean realmente nuevos diagnósticos. Se trataría de pacientes diagnosticados varios años antes, pero que no habrían vuelto a contactar con los servicios sanitarios debido a sus características sociales. Otro grupo podría estar constituido por 'antiguos' UDI, que usaron drogas por un período corto de tiempo y que estando asintomáticos han permanecido sin percepción de posible/probable infección.

El RD/PEA es más frecuente entre inmigrantes que entre la población española. Se conocen diferentes factores que favorecen la ausencia o el retraso de contacto de los inmigrantes con los servicios de salud: barreras lingüísticas, nivel sociocultural, situación administrativa, estigma social, desconocimientos de aspectos relacionados con la infección $\mathrm{VIH}^{28-29}$. Hemos encontrado un mayor riesgo de PEA en todas las áreas estudiadas (excluyendo América del Norte, Asia y Oceanía debido al escaso número nuevos diagnósticos) en relación con Europa Occidental.

La falta de disponibilidad del recuento de linfocitos CD4 en la totalidad de nuevos diagnósticos constituye una limitación a este estudio, no obstante el porcentaje de pacientes sin recuento de linfocitos CD4 es similar al de estudios realizados en nuestro medio y las conclusiones no difieren. El retraso en la notificación constituye otra limitación, si bien la cobertura del sistema de vigilancia está muy próxima al cien por cien y no es probable que 
la subnotificación influya en la información sobre el retraso diagnóstico.

Aunque los países del primer mundo disponen de medios para realizar diagnósticos con precocidad, los resultados que se desprenden de diferentes estudios, incluyendo el nuestro, evidencian que no son suficientes para reducir los diagnósticos tardíos. Deben intensificarse las herramientas existentes y crear otras nuevas en los casos, situaciones o escenarios que se estimen oportunos. Se deben considerar:

(1) Reforzar las actuaciones de prevención y promoción de salud dirigidas a la población, así como crear nuevas estrategias en la difusión de la información. Es necesario universalizar el conocimiento de las conductas de riesgo y, especialmente, sus consecuencias. Debe evitarse minimizar la gravedad de la infección por VIH.

(2) Facilitar la accesibilidad a la realización de las pruebas diagnósticas rápidas de infección por VIH, especialmente en los servicios de urgencias.

(3) Potenciar la creación y puesta en marcha de protocolos diagnósticos y terapéuticos que incluyan la realización de estudios diagnósticos de infección por VIH. La inclusión del estudio serológico en el protocolo de manejo del embarazo ha permitido la detección de infecciones desconocidas por las mujeres. El manejo clínico de ciertas patologías debe incluir la realización de una serología frente al VIH: infecciones de transmisión sexual, infecciones por virus hepatotropos, enfermedad tuberculosa, leishmaniasis visceral, infecciones neumocócicas, cuadros de herpes zoster, ciertos tumores sólidos, neumonía adquirida en la comunidad, etcétera y, en general, cualquier proceso cuya frecuencia esté aumentada en la población VIH-positiva.

(4) Desarrollar estrategias que incrementen la sospecha diagnóstica de infección por VIH entre los trabajadores de salud. En un número no despreciable de casos el diagnóstico de infección por VIH se realiza después de varios 'contactos' del paciente con el sistema de salud, como hemos comunicado recientemen$\mathrm{te}^{30}$.

En función de la prevalencia de infección por VIH, prevalencia oculta de infección por VIH y frecuencia del 'retraso diagnóstico' y 'presentación con enfermedad avanzada', podría valorarse la utilidad del cribado universal o no selectivo, siguiendo los criterios de Wilson y Jungner ${ }^{31}$. Se ha comunicado que esto sería especialmente recomendable y justificado en áreas donde la prevalencia de infección es superior al $0,1 \%{ }^{32-34}$. No obstante, los cribados selectivos parecen más justificados y más fácilmente abordables que los universales. Las definiciones de RD y PEA pueden facilitar la optimización de recursos, reforzar las estrategias existentes y favorecer el desarrollo de otras nuevas, adicionalmente la monitorización de los diagnósticos con menos de $500 \mathrm{CD} 4+/ \mu 1$ nos permitirá conocer el porcentaje de pacientes diagnosticados en el momento optimo de iniciar terapia ${ }^{16}$. Reducir al máximo el RD y, sobre todo, la PEA es una exigencia clínica y epidemiológica y constituye un reto en salud pública y debe formar parte de cualquier política sanitaria.

\section{AGRADECIMIENTOS}

Queremos expresar nuestra gratitud a todos los profesionales implicados en el diagnóstico, tratamiento y prevención del VIH/sida en la Comunidad de Madrid por su trabajo en el mantenimiento y actualización del registro sin el cual no se hubiese podido elaborar este estudio.

\section{BIBLIOGRAFÍA}

1. Girardi E, Sabin CA, d'Arminio A. Late Diagnosis of HIV Infection: Epidemiological Features, Consequences and Strategies to Encourage Earlier Testing. J Acquir Immune Defic Syndr. 2007; 46 Sup13: S3-S8

2. Egger M, May M, Chêne G, Phillips AN, Ledergerber B, Dabis F et al. Prognosis of HIV-1-infected patients starting highly active antiretroviral therapy: a collaborative analysis of prospective studies. Lancet. 2002; 360:119-29. 
3. Chadborn TR, Baster K, Delpech VC, Sabin CA, Synka $\mathrm{K}$, Rice BD et al. No time to wait: how many HIV-infected homosexual men are diagnosed late and consequently die? (England and Wales, 1993-2002). AIDS. 2005;19: 513-20.

4. Marks G., Crepaz N., Jansenn RS. Estimating sexual transmission of HIV from persons aware and unaware that they are infected with the virus in the USA. AIDS. 2006: 20: $1447-50$

5. Krentz HB, Auld MC, Gill MJ. The high cost of medical care for patients who present late (CD4 <200 cells/microL) with HIV infection. HIV Med. 2004; 5:938.

6. Klein D, Hurley LB, Merrill D, Quesenberry CP. Consortium for HIV/AIDS Interregional Research. Review of medical encounters in the 5 years before a diagnosis of HIV-1 infection: implications for early detection. J Acquir Immune Defic Syndr. 2003; 32: 142-52

7. Manavi K, McMillan A, Ogilvie M, Scott G. Heterosexual men and women with HIV test positive at a later stage of infection than homo- or bisexual men. Int J STD AIDS. 2004;15:811-4.

8. Girardi E, Aloisi MS, Arici C, Pezzotti P, Serraino D, Balzano R, et al. For the IcoNA Behavioural Epidemiology Study Group. Delayed presentation and late testing for HIV: demographic and behavioral risk factors in a multicenter study in Italy. J Acquir Immune Defic Syndr.2004; 36:951-9.

9. Sullivan AK, Curtis H, Sabin CA, Johnson MA. Newly diagnosed HIV infections: review in UK and Ireland. BMJ. 2005; 330: 1301-2

10. Antinori A, Coenen T, Costagiola D, Dedes N, Ellefson M, Gatell J, et al. European Late Presenter Consensus Working Group. Late presentation of HIV infection: a consensus definition. HIV Med. 2011;12: 61-4

11. UK Collaborative HIV Cohort (UK CHIC) Steering Committee, Sabin CA, Schwenk A, Johnson MA, Gazzard B, Fisher M, Walsh J, et al. Late diagnosis in the HAART era: proposed common definitions and associations with mortality. AIDS. 2010; 24:723-7.

12. Verdejo J, Cevallos C, Izarra C, Martínez-Rodríguez S. Retraso diagnóstico de la infección por VIH en la Comunidad de Madrid. XXIX Reunión Científica de la Sociedad Española de Epidemiología. XIV Congreso de la Sociedad Española de Salud Pública y Administración Sanitaria. Madrid: XXIX Reunión Científica de la Sociedad Española de Epidemiología. XIV Congreso de la Sociedad Española de Salud Pública y Administración Sanitaria. Gac Sanit. 2011; 25.340.
13. Sistema de información de nuevas infecciones de VIH en Aragón (SINIVIH) Año 2009. Registro de casos de sida de Aragón. Servicio de Vigilancia de Salud Pública. [Citado mayo 2011]. Disponible en: https://www.aragon.es/estaticos/GobiernoAragon/De partamentos/SaludConsumo/Documentos/docs2/Profesionales/Salud\%20publica/Vigilancia \%20epidemiol $\%$ C3\%B3gica/Enfermedades $\% 20$ Declaraci $\% \mathrm{C3}$ $\%$ B 3 n $\% 20$ Obligatoria $\% 20$ otros $\% 20$ procesos/20091230\%20Informe\%20VIH.pdf.

14. Panel on Antiretroviral Guidelines for Adults and Adolescents. Guidelines for the use of antiretroviral agents in HIV-1-infected adults and adolescents. Department of Health and Human Services, January 10, 2011; 1-166. Disponible en http://www.aidsinfo.nih.gov/ContentFiles/AdultandAdolescentGL.pdf Revisado Mayo 2011

15. Thompson MA, Aberg JA, Cahn P, Montaner JS, Rizzardini $G$, Telenti A, et al. International AIDS Society-USA. Antiretroviral treatment of adult HIV infection: 2010 recommendations of the International AIDS Society-USA panel. JAMA. 2010; 304: 321-33.

16. Documento de consenso de Gesida/Plan Nacional sobre el Sida respecto al tratamiento antirretroviral en adultos infectados por el virus de la inmunodeficiencia humana (Actualización enero 2012). [Citado en febrero 2012 ]. Disponible en: http://www.msssi.gob.es/ciudadanos/enfLesiones/enf Transmisibles/sida/publicaciones/profSanitarios/Con sensoTARGESIDAPNS2012_ordenador.pdf.

17. Charlebois ED, Das M, Porco TC, Havlir DV. The Effect of Expanded antiretroviral Treatment Strategies on the HIV Epidemic among Men Who Have Sex with Men in San Francisco. Clin Infect Dis 2011; 52:1046-9

18. Diario Oficial de la Unión Europea. Decisión de la Comisión de 28 de abril de 2008 por la que se establecen las definiciones de los casos para comunicar las enfermedades transmisibles a la red comunitaria, de conformidad con la Decisión núm 2119/98/CE del Parlamento Europeo y del Consejo. DOCE de $18 / 6 / 2008$

19. Boletín Oficial de la Comunidad de Madrid. ORDEN 372/2010, de 15 de julio, por la que se modifica el sistema de notificación de enfermedades de declaración obligatoria por infección por virus de inmunodeficiencia humana (VIH). BOCM Num. 186 de $5 / 8 / 2010$

20. Althoff KN, Gange SJ, Klein MB, Brooks JT, Hogg RS, Bosch RJ, et al. Late presentation for human immunodeficiency virus care in the United States and Canada Clin Infect Dis. 2010; 50:1512-20. 
21. Díez M, Oliva J, Sánchez F, Vives N, Cevallos C, Izquierdo A por el Grupo SINIVH. Incidencia de nuevos diagnósticos de VIH en España, 2004-2009. Gac Sanit. (en prensa). doi: 10.1016/j.gaceta.2011.07.023.

22. Sobrino-Vegas P, García-San Miguel L, Caro-Murillo AM, Miró JM, Viciana P, Tural C. et al.. Delayed Diagnosis of HIV Infection in a Multicenter Cohort: Prevalence, Risk, Factors, Response to HAART and Impact on Mortality. Curr HIV Res. 2009 Mar;7(2):224-30

23. Oliva J, Galindo S, Vives N, Arrillaga A, Izquierdo A, Nicolau A, et al. Retraso diagnóstico de la infección por el virus de la inmunodeficiencia humana en España Enferm Infecc Microbiol Clin. 2010; 28: 583-9

24. Sobrino P, del Amo J, Santos I, Riera M, del Romero J, Fiorante $\mathrm{S}$ et al. Magnitud y factores asociados al diagnóstico tardío de la infección por VIH en la cohorte CORIS.. XXIX Reunión Científica de la Sociedad Española de Epidemiología. XIV Congreso de la Sociedad Española de Salud Pública y Administración Sanitaria. Madrid. Gac Sanit. 2011; 25:339.

25. European Centre for Disease Prevention and Control/WHO Region Office for Europe. HIV/AIDS surveillance in Europe 2010. Stockholm: European Centre for Disease Prevention and Control; 2011

26. Garcia de Olalla P, Manzardo C, Sambeat MA, Ocaña I, Knobel H, Humet V et al. Epidemiological Characteristics and predictors of late presentation of HIV infection in Barcelona (Spain) during the period 2001-2009. AIDS Res Ther. 2011 Jul 6;8(1):22.

27. Mugavero MJ, Castellano C, Edelman D, Hicks C. Late diagnosis of HIV infection: the role of age and sex. Am J Med. 2007; 120:370-3.

28. Levy V, Prentiss D, Balmas G, Chen S, Israelski D, Katzenstein D, et al. Factors in the delayed HIV presentation of immigrants in Northern California: implications for voluntary counseling and testing programs. J Immigr Minor Health. 2007; 9: 49-54

29. La prevención de la infección del VIH/sida en la población inmigrante. Ministerio de Sanidad y Consumo 2006. Disponible en :

http:/www.mspsi.es/ciudadanos/enfLesiones/enfTransmisibles/sida/prevencion/inmigrantes/docs/prevencionVIH_SIDAPoblacionInmigrante06.pdf. Revisado Mayo 2011

30. Moreno S, Ordobas M, Sanz JC, Ramos B, Astray J, Ortiz M, et al. Prevalence of Undiagnosed HIV-Infection in the general population in Madrid, Spain: Evidence for the need of effective testing programs. Vienna: XVIII International AIDS Conference; 2010 (July, 18-23)
31. Wilson JMG, Jungner G. Principles and Practice of Screening for Disease. Geneva: World Health Organization; 1968. Disponible en :

http://whqlibdoc.who.int/php/WHO_PHP_34.pdf. Revisado Mayo 2011

32. Branson BM, Handsfield HH, Lampe MA, Janssen RS, Tylor AW, Lyss SB et al. Centers for Disease Control and Prevention (CDC) Revised recommendations for HIV testing of adults, adolescents, and pregnant women in health-care. MMWR Recomm Rep. 2006; 55(RR-14):117

33. Brown J, Shesser R, Simon G, Bahn M, Czarnogorski M, Kuo I, et al. Routine HIV screening in the emergency department using the new US Centers for Disease Control and Prevention Guidelines: results from a high-prevalence area. J Acquir Immune Defic Syndr. 2007; 46:395-401.

34. UK national guidelines for HIV testing 2008. [Citado mayo 2011]. Disponible en:

http://www.guideline.gov/content.aspx?id=14297 